\title{
Perceived Maternal Autonomy Support and Early Adolescent Emotion Regulation: A Longitudinal Study
}

\author{
Katrijn Brenning, Bart Soenens, Stijn Van Petegem, \\ Maarten Vansteenkiste, Ghent University
}

\begin{abstract}
This study investigated longitudinal associations between perceived maternal autonomy-supportive parenting and early adolescents' use of three emotion regulation (ER) styles: emotional integration, suppressive regulation, and dysregulation. We tested whether perceived maternal autonomy support predicted changes in ER and whether these ER styles, in turn, related to changes in adjustment (i.e., depressive symptoms, self-esteem). Participants $(N=311$, mean age at Time $1=12.04)$ reported on perceived maternal autonomy support, their ER styles, and adjustment at two moments in time, spanning a one-year interval. Cross-lagged analyses showed that perceived maternal autonomy support predicted increases in emotional integration and decreases in suppressive regulation. By contrast, emotional dysregulation predicted decreases in perceived autonomy-supportive parenting. Further, increases in emotional integration were predictive of increases in self-esteem, and decreases in suppressive regulation were predictive of decreases in depressive symptoms. Together, the results show that early adolescents' perception of their mothers as autonomysupportive is associated with increases in adaptive ER strategies and subsequent adjustment.
\end{abstract}

Keywords: autonomy support; parenting; emotion regulation; longitudinal

\section{Introduction}

Throughout childhood and early adolescence, children become increasingly independent in regulating and managing their emotions (Gross, 2013; Morris, Silk, Steinberg, Myers, \& Robinson, 2007; Zeman, Cassano, Perry-Parrish, \& Stegall, 2006). This development is fostered by children's emerging capacity to use more sophisticated and constructive emotion regulation (ER) styles (McRae et al., 2012; Zeman et al., 2006). Still, the development of adequate ER skills is far from finished by the end of

Katrijn Brenning is a postdoctoral researcher at the Special Research Fund of Ghent University (BOF).

Correspondence should be addressed to Katrijn Brenning, Department of Developmental, Personality and Social Psychology, Ghent University, H. Dunantlaan 2, 9000 Ghent, Belgium. Email: katrijn.brenning@ugent.be 


\section{Katrijn Brenning, Bart Soenens, Stijn Van Petegem et al.}

childhood or early adolescence. Adequate regulation of emotions may even become more challenging due to rapid social and biological changes. For instance, because of heightened stress reactivity evidenced in the hypothalamic-pituitary-adrenal (HPA) axis and other neurobiological systems, young adolescents may respond more intensively to negative emotions (e.g., Romeo, 2013).

During this period, parents may play an important role in the development of adaptive ER styles (Morris et al., 2007). Parents can affect their children's ER strategies in several ways (Morris et al., 2007). For instance, they can serve as a role model or provide guidance concerning the management of emotions in specific situations. One important line of research regarding parents' direct coaching of emotions is inspired by meta-emotion theory (Gottman, Katz, \& Hooven, 1997). In this theory, a distinction is made between emotion-coaching parenting and emotion-dismissive parenting. In the case of emotion-coaching parenting, parents treat the emotion expressed by the child as useful information, thereby conveying the message that the emotion is acceptable. Specifically, parents are aware of the child's emotions, empathize with negative emotions, help the child verbally label emotions, and communicate about family values. In the case of emotion-dismissive parenting, parents display an opposite set of responses, thereby communicating that certain emotions are unacceptable. Specifically, parents are unsufficiently aware of the child's emotions, use impoverished emotion vocabulary, and try to alter the child's emotions instead of using the opportunity to teach new ER skills to the child. Whereas emotion-coaching parenting has been found to relate to more adaptive outcomes in children (e.g., academic adjustment, impulse control, psychosocial well-being), emotion-dismissive parenting was associated with more maladaptive outcomes (e.g., Dunsmore, Booker, \& Ollendick, 2013; Gottman et al., 1997). These findings are consistent with research conducted within the context of emotion socialization theory (e.g., Fabes, Leonard, Kupanoff, \& Martin, 2001), showing that parental socialization behaviors, such as expressive validation responses (akin to emotion-coaching parenting), are linked to emotional competence. By contrast, practices such as minimization of children's emotional displays (akin to emotion-dismissing parenting) were related to indicators of emotional incompetence.

In addition to these relatively direct ways of being involved with children's ER, it has been argued that the general affective tone and quality of parents' rearing style may also be important. According to Morris et al. (2007), general parenting style is an important factor in the development of ER. As noted by Morris et al. (2007, p. 371), 'there is little research on the impact of parenting styles on adolescents' ER and longitudinal studies examining links between parenting and ER over time are greatly needed'. The present longitudinal study aimed to heed this call by addressing the role of perceived maternal autonomy support in early adolescent ER. Autonomy-supportive parenting is a dimension of general parenting style that has not been examined systematically in relation to ER processes.

\section{Autonomy-supportive Parenting}

Autonomy support is conceptualized in this study from the perspective of selfdetermination theory (SDT; Deci \& Ryan, 2000). SDT is a macro-theory on motivation and social development that posits three fundamental psychological human needs as vital ingredients for children's growth and development, that is, the needs for relatedness (i.e., experiencing intimacy and caring in close relationships), competence (i.e., experiencing a sense of effectiveness), and autonomy (i.e., experiencing personal 
choice and volition in one's actions). These needs are nurtured when parents adopt a need-supportive style, which involves (1) structure (e.g., communicating clear and consistent guidelines for behavior), (2) involvement (e.g., creating a warm and responsive climate in the interaction with the child), and (3) autonomy support (Joussemet, Landry, \& Koestner, 2008; Soenens \& Vansteenkiste, 2010).

Parental autonomy support is a fairly stable dimension of parenting (Matte-Gagne, Bernier, \& Gagne, 2013) characteristic of parents who encourage their children to behave upon self-endorsed values and preferences (Soenens et al., 2007). Three key strategies of autonomy-supportive parenting have been studied most intensively. The first element is empathy, which involves trying to relate to and understand the child's perspective. Second, autonomy-supportive parents provide meaningful choices whenever possible. Third, when choice is limited, autonomy-supportive parents offer a meaningful rationale.

Various studies with adolescent samples have shown that perceived autonomy support is conducive to adaptive developmental outcomes, such as psychological well-being, academic engagement, and attachment to parents (Brenning, Soenens, Braet, \& Bosmans, 2012; Joussemet et al., 2008; Roth, Assor, Niemiec, Ryan, \& Deci, 2009). Although this growing body of work underscores the importance of parental autonomy-supportive behaviors for adolescents' psychosocial development, few studies to date have examined the consequences of autonomy support for adolescents' ER. As processes of ER have important repercussions for development and adjustment (e.g., Zeman et al., 2006), ER could be one key component that links autonomy support to adolescent adjustment.

\section{Distinguishing between Different Styles of ER}

ER refers to the various conscious and unconscious processes by which emotions themselves are regulated (Gross, 2013). Several frameworks describe individual differences in ER styles. In this study, we used the perspective of SDT on adolescent and adult ER, where a distinction is made between three ER styles that differ in terms of the quality and depth of processing and regulating emotions (Roth et al., 2009).

Emotional integration is considered the most adaptive style of ER. It is characterized by openness to experience emotions in full awareness and to explore emotions in a thorough and unbiased way. Children high on integrative regulation have a genuine interest in their inner emotional world. They allow their emotions to emerge and to be truly felt without trying to hide them. Due to the receptivity and acceptance of emotions, emotions can be used in an informational way; that is, they can serve as a guide for subsequent behavior. Children high in integrative regulation flexibly use a wide range of possible strategies. For instance, emotional integration may involve either the expression or the withholding of the felt emotions. Yet, in both cases, individuals deliberately choose to show their emotions or to keep them for themselves. Research with both middle childhood-aged children and adolescents (Roth \& Assor, 2012) showed that emotional integration is related to adaptive outcomes.

By contrast, suppressive regulation and dysregulation are considered suboptimal ER styles because they represent defensive ways of regulating negative emotions. Suppressive regulation involves the avoidance or minimization of emotional experiences. Children high on suppressive regulation feel like there is no other way to regulate their emotions but to suppress them. Emotional suppression may seem to have some shortterm adaptive value as children would not be overwhelmed by negative emotions. Yet, 


\section{Katrijn Brenning, Bart Soenens, Stijn Van Petegem et al.}

in the longer run, suppression may be disabling because of its relatively controlling character. That is, when displaying suppression, children feel pressured to alter, downplay, or even dismiss their negative emotions, such that these emotions cannot be experienced as such.

Dysregulation involves a poorly deployed range of strategies that are ineffective at downregulating negative emotions. Children high on dysregulation tend to feel overwhelmed by their emotions, such that their emotions are expressed in their behavior unintentionally and such that the expression of emotions is often extreme and intense. Dysregulation would be harmful because it involves helplessness and a lack of control of negative emotions. Every overwhelming experience of negative emotions will interfere further with children's confidence to adequately cope with subsequent negative emotions (Cicchetti, Ackerman, \& Izard, 1995).

Consistent with the notion that suppression and dysregulation represent less healthy ways of ER, they have been found to be related to suboptimal functioning and ill-being (Gross, 2013). For example, in research with middle childhood-aged children and adolescents, both ER styles independently predicted more depressive symptoms (Brenning et al., 2012) and lower quality of interpersonal functioning (Roth \& Assor, 2012).

\section{Perceived Autonomy-supportive Parenting and ER}

According to SDT, autonomy-supportive parents display an active and authentic interest in children's (negative) emotions. Specifically, autonomy-supportive parents create an open atmosphere in which children's emotions are accepted as they are. Further, autonomy-supportive parents encourage children to explore their emotions, and if desired allow them to express and discuss these emotions (Roth et al., 2009). In doing so, they try to respect the timing and pace of children's emotional disclosure, thereby refraining from pushing their children to express their negative emotions. Parents' acknowledgment of children's feelings in combination with the provision of desired guidance in handling negative emotions might create opportunities for children to allow, explore, and reflect upon negative emotions, and to respond to those emotions in a non-defensive and integrative fashion (Roth \& Assor, 2012).

In spite of the theoretical arguments for the role of autonomy-supportive parenting in adolescents' ER styles, few studies to date explicitly address the role of autonomy support. In a number of cross-sectional studies with middle childhood-aged children and adolescents (e.g., Brenning et al., 2012; Roth \& Assor, 2012; Roth et al., 2009), perceived parental autonomy support related positively to adaptive ER (i.e., integration) whereas a perceived autonomy-suppressing parental style related positively to maladaptive ER (i.e., suppression and dysregulation). Yet previous research is limited in two ways, which we aim to overcome in the present study. First, there is a dearth of longitudinal research addressing the direction of effects. Although autonomy support is expected to be predictive of children's development of ER, associations between parenting and ER may also be bidirectional. For instance, being autonomy-supportive likely is easier for parents when children display adaptive styles of ER. Second, it is unclear whether these ER styles explain why autonomy-supportive parenting is related to adolescent adjustment. Hence, we also aimed to test for the role of ER styles as an intervening variable in the association between parental autonomy support and adjustment. 


\section{The Present Study}

This study examined longitudinal associations between perceived maternal autonomy support, different styles of ER (i.e., emotional integration, suppressive regulation and dysregulation) and adjustment in early adolescents. We measured ER styles specifically with regard to sadness because sad emotions are particularly prevalent during early adolescence (e.g., Larson, Moneta, Richards, \& Wilson, 2002) and because adolescents' ER styles for sadness are strongly predictive of depressive symptoms (e.g., Brenning \& Braet, 2013). We included depressive symptoms as a key outcome in the present study as it is a salient and common manifestation of psychopathology in early adolescence (e.g., Kessler, Avenevoli, \& Merikangas, 2001). Further, we included self-esteem because it represents an adaptive developmental outcome that has been found to be related to parental autonomy support (e.g., Soenens \& Vansteenkiste, 2005). We hypothesized that maternal autonomy-supportive parenting would predict increased use of integrative ER whereas it would predict decreases in suppressive and dysregulated ER styles. We also tested whether ER styles predicted changes in perceived autonomy support. For example, when children explore their own sad emotions, thereby disclosing them in a volitional way (which characterizes integration), it might be easier for mothers to understand and take the child's perspective. By contrast, both a secretive and inhibited orientation (characteristic of suppression) and an emotionally disorganized orientation (characteristic of dysregulation) may elicit annoyance or stress in mothers, such that mothers respond in a less autonomy-supportive fashion. Further, we hypothesized that integrative ER would be associated with adaptive outcomes (i.e., high self-esteem and low depressive symptoms); suppressive and dysregulative ER styles would show an opposite pattern of associations. Finally, we investigated the intervening role of ER in associations between maternal autonomy support and adjustment.

\section{Method}

\section{Participants and Procedure}

Data of Wave 1 from this two-wave longitudinal study were gathered in the context of a course on developmental psychology. Undergraduate students were asked to invite two non-related children in the Dutch-speaking part of Belgium to participate in this study. They visited the participants at home in order to hand them a set of paper and pencil questionnaires. This was done after active parental consent was obtained for the child's participation. Participation in the study was voluntary and confidentiality was guaranteed. All participants were assigned a unique code to ensure confidential treatment of the data. About one year later (with a maximum range of 1 month), a different group of students in the context of the same course on developmental psychology invited participants by phone to fill out the same questionnaires again. When both parents and children agreed, the questionnaires were sent to the participants by mail. Ethical approval for this study was granted by the organizing university's institutional review board.

This procedure resulted in a sample of 311 Dutch-speaking Belgian pre- to early adolescents (53.7 percent female), with a mean age at Time 1 of 12.04 years (ranging between 9 and 14 years; $S D=1.41)$. Two hundred sixty-five participants ( 85.2 percent) came from intact families. Regarding level of education, 77 percent of the participants 


\section{Katrijn Brenning, Bart Soenens, Stijn Van Petegem et al.}

were following the academic track (i.e., preparing for college or university studies) whereas the remaining participants were preparing for technical proficiencies. Of the 311 children participating, 168 (54 percent) participated at both measurement times. Cases with missing values at Time $1(\mathrm{~N}=22)$ or at Time $2(\mathrm{~N}=121)$ were included in the analyses using full-information maximum likelihood estimation (FIML; Little \& Rubin, 1987). As FIML only can be used when no systematic differences are found between participants who stay in vs. participants who drop out, both subsamples were compared on the study variables. Using one-way analyses of variance, no significant differences were found between children who participated twice and children who participated only once on any of the study variables (mean $F=.64, p>.05$ ). Further, Little's missing completely at random (MCAR) test produced a normed $\chi^{2}\left(\chi^{2} / d f\right)$ of 1.27 , which indicates that the data were likely to be missing at random, and that it is safe to include cases with missing values in the analyses.

\section{Measures}

Perceived Autonomy Support. Seven items from the autonomy support subscale of the perceptions of parents scale (Grolnick, Ryan, \& Deci, 1991) were used to capture perceptions of maternal autonomy support. A sample item reads: 'My mother, whenever possible, allows me to choose what to do'. Participants rated the items on a scale from 1 (strongly disagree) to 5 (strongly agree). The psychometric quality and validity of the scale are well established (Soenens et al., 2007). In the present study, Cronbach's alpha was .64 at Time 1 and .72 at Time 2 .

ER. ER styles were assessed using the emotion regulation inventory (ERI) developed by Roth et al. (2009) for use with both adolescents and adults. The ERI with regard to sad emotions contains three subscales: integration (six items, e.g., 'Feelings of sadness can sometimes help me understand important things about myself'), suppression (six items, e.g., 'When I feel sad, I almost always hide it so others won't notice it'), and dysregulation (six items, e.g., 'It is hard for me to control my sad emotions'). All items were rated on a scale from 1 (completely disagree) to 5 (completely agree). Previous research has provided evidence for the internal structure and validity of this instrument (see, e.g., Brenning et al., 2012). Although the present study is the first to use the scale for integration with regard to sadness in particular, Brenning et al. (2012) have shown that dysregulation and suppression correlated in theoretically plausible ways with measures of attachment anxiety and avoidance. In the current study, Cronbach's alphas for integration, suppression, and dysregulation were, respectively, .62, .80, and .64 at Time 1 , and .68, .81, and .72 at Time 2 .

Depressive Symptoms. The children's depression inventory (Kovacs, 1985) aims to measure depressive symptoms from 7 to 17 years of age. This self-report scale includes 27 items dealing with sadness, self-blame, loss of appetite, insomnia, and interpersonal relationships. Each item is scored on a scale from 0 (symptom is absent) to 2 (symptom is present most or all the time). Acceptable levels of internal consistency, validity, and test-retest reliability have been established (e.g., Kovacs, 1985). In the current study, Cronbach's alphas were .79 at Time 1 and .80 at Time 2. Elevated levels of depressive symptoms, indicated by a minimum score of 16 (Timbremont, Braet, \& Roelofs, 2008), were reported by 8.7 percent of the participants at Time 1 and by 9.3 percent of the participants at Time 2 . 
Self-Esteem. Participants completed the six-item global self-worth subscale of the self-perception profile for children (Harter, 1985). The questionnaire was modified with respect to its item format. In the original format, participants were asked to make a choice between two statements, each describing an adolescent with opposite characteristics. As proposed by Wichstrom (1995), we used the more straightforward item format, in which only one statement is used for each item. In the current study, Cronbach's alpha was .84 at Time 1 and .86 at Time 2.

\section{Results}

\section{Descriptive Statistics and Preliminary Analyses}

Correlations among the study variables are presented in Table 1. All variables demonstrated substantial rank-order stability from Time 1 to Time 2. The strongest stability coefficient was found for autonomy support, followed by depressive symptoms and self-esteem. The ER strategies were relatively less stable. As expected, perceived autonomy support correlated negatively with depressive symptoms and positively with self-esteem, both across and within measurement waves. Further, both across and within waves, autonomy support related positively to integrative ER and negatively to suppressive ER. Correlations between autonomy support and emotional dysregulation were all negative both across and within measurement waves, but were not consistently significant. Finally, correlations between the different ER strategies and adjustment were in the expected direction, with integration relating positively to self-esteem both across and within measurement waves, and more maladaptive ER strategies (i.e., suppression and dysregulation) relating positively to depressive symptoms both across and within measurement waves. Table 1 also presents the means and standard deviations of all study variables.

Next, preliminary analyses were conducted to examine differences in the study variables in terms of age, gender, and family structure. A first multivariate analysis of covariance (MANCOVA) (with age as a covariate and with gender and family structure as fixed factors) examined effects on perceived maternal autonomy support at Time 1 and Time 2. No significant multivariate effects were obtained for age, Wilks' lambda $=$ $.99, F(2,158)=1.13, p>.05$; gender, Wilks' lambda $=.97, F(2,158)=2.52, p>.05$; or family structure, Wilks' lambda $=.99, F(2,158)=1.09, p>.05$. A second MANCOVA examined effects on the three ER scales. Again, no significant multivariate effects were obtained for age, Wilks' lambda $=.93, F(6,153)=2.06, p>.05$; gender, Wilks' lambda $=.94, F(6,153)=1.78, p>.05$; or family structure, Wilks' lambda $=.96, F(6,153)=$ $1.18, p>.05$. A third MANCOVA examined effects on depressive symptoms and self-esteem. A significant multivariate effect was obtained for age, Wilks' lambda $=.91$, $F(4,154)=3.70, p<.01$. The results showed a significant association between age and both self-esteem (Time 1: $r=-.15, p<.05$ and Time $2: r=-.30, p<.001$ ) and depressive symptoms (Time $2: r=.20, p<.01$ ). No significant multivariate effects were obtained for gender, Wilks' lambda $=.96, F(4,154)=1.44, p>.05$ or family structure, Wilks' lambda $=.97, F(4,154)=1.42, p>.05$. Based on these preliminary analyses, age was included as a control variable in all subsequent analyses.

\section{Primary Analyses}

To examine the main hypotheses, cross-lagged analyses with latent variables were conducted using Mplus (Muthén \& Muthén, 2012). An important advantage of this 
568 Katrijn Brenning, Bart Soenens, Stijn Van Petegem et al.

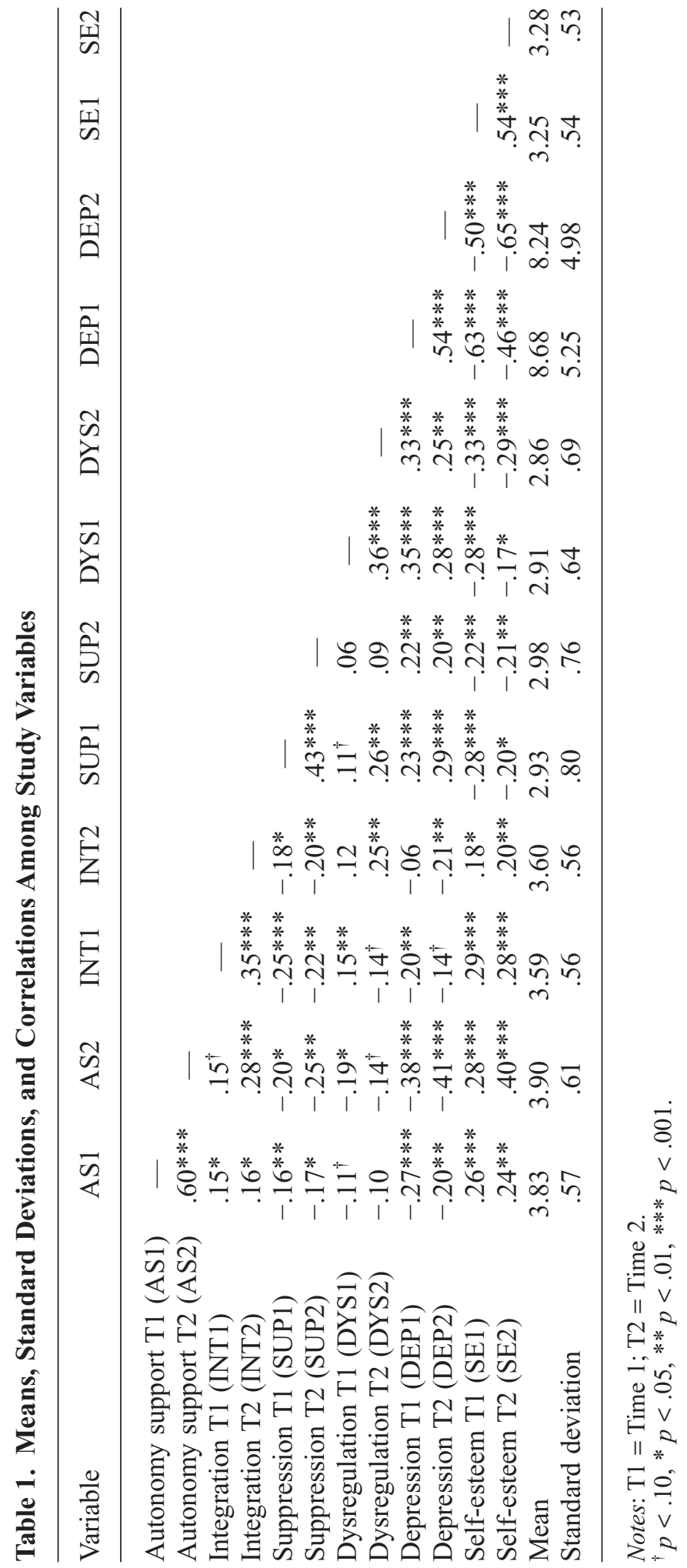


approach is that it controls for measurement error (which is important in the current study as some of the Cronbach's alpha values were modest) and that it allows one to examine measurement equivalence across waves prior to examining structural associations. For each construct, three randomly created parcels were computed, and the same parceling procedure was used at Time 1 and Time 2. All structural cross-lagged path analyses contained (1) stability coefficients of the constructs, (2) within-time associations between the constructs, and (3) cross-lagged paths between each of the constructs across time. We evaluated model fit based on a combined consideration of the chi-square statistic $\left(\chi^{2}\right)$, the comparative fit index (CFI), the root mean square error of approximation (RMSEA), and the standardized root mean square residual (SRMR). The $\chi^{2}$ should be as small as possible. A CFI value of .90 or higher indicates a reasonable fit whereas an RMSEA value of .06 or lower and an SRMR value of .08 or lower indicate acceptable fit (Kline, 2010). Before testing the structural models, we conducted a multigroup confirmatory factor analysis to assess the longitudinal invariance of the measurement model.

Measurement Model. The baseline model included 12 latent variables (i.e., perceived autonomy support, three ER styles, and two outcomes, each assessed at two measurement points) and 36 indicators (i.e., three parcels for each latent construct). The measurement errors of the same indicators at different measurement points were allowed to covary. This model fitted the data adequately, $\chi^{2}(510)=808.12, \mathrm{CFI}=.91$, $\mathrm{RMSEA}=.04, \mathrm{SRMR}=.06 . \mathrm{Next}$, a model was estimated in which the factor loadings were set equal across the two measurement points. Compared with the model with freely varying factor loadings, the latter model did not result in a significant loss in model fit, $\Delta \chi^{2}(12)=9.40, p>.05$. Moreover, all factor loadings were highly significant $(p<.001)$, ranging from .28 to 1.00 (mean $=.70)$. In sum, evidence was obtained for a reliable and longitudinally invariant measurement model, which was used in all subsequent tests of the structural models.

Structural Models. Prior to testing integrated structural models, we tested a series of simpler cross-lagged models to examine the direction of effects involved in associations between all study variables.

Perceived autonomy support and adjustment. A first cross-lagged model involved associations between perceived autonomy-supportive parenting and depressive symptoms, $\chi^{2}(57)=119.24, \mathrm{CFI}=.93, \mathrm{RMSEA}=.06, \mathrm{SRMR}=.07$. Maternal autonomy support was predictive of decreases in depressive symptoms across time $(\beta=-.20$, $p<.05)$. Conversely, depressive symptoms had a marginally significant effect on decreases in autonomy support $(\beta=-.16, p=.07)$. A second cross-lagged model involved the bidirectional associations between autonomy-supportive parenting and self-esteem, $\chi^{2}(56)=79.55, \mathrm{CFI}=.98, \mathrm{RMSEA}=.04, \mathrm{SRMR}=.06$. Autonomy support predicted increases in self-esteem $(\beta=.18, p<.05)$ whereas self-esteem did not predict changes in autonomy support $(\beta=.03, p>.10)$.

Perceived autonomy support and ER. A second set of cross-lagged models tested longitudinal associations between perceived maternal autonomy support and the ER styles. A first cross-lagged model involved autonomy support and emotional integration, $\chi^{2}(57)=78.45, \mathrm{CFI}=.96, \mathrm{RMSEA}=.04, \mathrm{SRMR}=.06$. This model showed a marginally significant effect of autonomy-supportive parenting on increases in 
integration $(\beta=.19, p=.07)$ whereas integration did not predict significant changes in autonomy-supportive parenting $(\beta=-.05, p>.10)$. A second cross-lagged model tested longitudinal associations between autonomy support and suppression, $\chi^{2}(58)=$ $69.87, \mathrm{CFI}=.99, \mathrm{RMSEA}=.03, \mathrm{SRMR}=.05$. Autonomy support predicted decreases in suppression $(\beta=-.24, p<.01)$, but suppression did not predict significant changes in autonomy support $(\beta=-.05, p>.10)$. A third model investigated the bidirectional associations between autonomy support and dysregulation, $\chi^{2}(58)=87.12, \mathrm{CFI}=.95$, $\mathrm{RMSEA}=.04, \mathrm{SRMR}=.06$. Autonomy support at Time 1 did not predict significant changes in dysregulation across time $(\beta=-.04, p>.10)$. However, initial levels of dysregulation significantly predicted decreases in autonomy support $(\beta=-.15$, $p=.05)$.

ER and adjustment. A third set of cross-lagged models tested longitudinal associations between the ER styles and adjustment. A first cross-lagged model involved emotional integration and depressive symptoms, $\chi^{2}(57)=99.50, \mathrm{CFI}=.95, \mathrm{RMSEA}=$ $.05, \mathrm{SRMR}=.06$. This model indicated that integration did not predict changes in depressive symptoms $(\beta=-.08, p>.10)$, nor did depressive symptoms predict changes in integration $(\beta=-.03, p>.10)$. A second model tested longitudinal associations between integration and self-esteem, $\chi^{2}(56)=90.22, \mathrm{CFI}=.97, \mathrm{RMSEA}=.04$, SRMR $=.08$. This model yielded evidence for bidirectional associations, with integration predicting increases in self-esteem $(\beta=.18, p<.05)$ and self-esteem predicting increases in integration $(\beta=.19, p<.05)$. A third model involved suppression and depressive symptoms, $\chi^{2}(58)=103.43, \mathrm{CFI}=.96$, RMSEA $=.05, \mathrm{SRMR}=.06$. This model showed that suppression predicted increases in depressive symptoms $(\beta=.24$, $p<.01)$ and showed a marginally significant effect of depressive symptoms on increases in suppression $(\beta=.15, p=.08)$. Further, no evidence was found for bidirectional associations between suppression and self-esteem, $\chi^{2}(56)=55.42, \mathrm{CFI}=$ $1.00, \mathrm{RMSEA}=.00, \mathrm{SRMR}=.04$. Specifically, suppression did not predict changes in self-esteem $(\beta=-.07, p>.10)$ or vice versa $(\beta=-.08, p>.10)$. A fifth model tested the longitudinal associations between dysregulation and depressive symptoms, $\chi^{2}(56)$ $=116.38, \mathrm{CFI}=.93$, RMSEA $=.06$, SRMR $=.06$. This model showed that dysregulation did not predict significant changes in depressive symptoms $(\beta=.08$, $p>.10)$. However, depressive symptoms did predict increases in dysregulation $(\beta=.29, p<.01)$. Finally, in the sixth model, we tested for the longitudinal associations between dysregulation and self-esteem, $\chi^{2}(56)=60.17$, CFI $=1.00$, RMSEA $=.02$, SRMR $=.04$. These analyses yielded a similar pattern. Specifically, dysregulation did not predict changes in self-esteem $(\beta=-.02, p>.10)$ whereas self-esteem did predict decreases in dysregulation $(\beta=-.29, p<.001)$.

In sum, based on a series of cross-lagged models, we found that perceived autonomy support more consistently predicted changes in adjustment than the other way around. Further, autonomy support predicted changes in both integration and suppression whereas an opposite direction of effects was found for dysregulation. Regarding the link between ER and adjustment, integration was related reciprocally to self-esteem, suppression was related reciprocally to depressive symptoms, and both depressive symptoms and self-esteem were predictive of changes in dysregulation.

Integrated model. Based on the results of the cross-lagged models presented so far, we tested several integrated models examining associations between perceived maternal autonomy support, styles of ER, and outcome variables. All significant and 


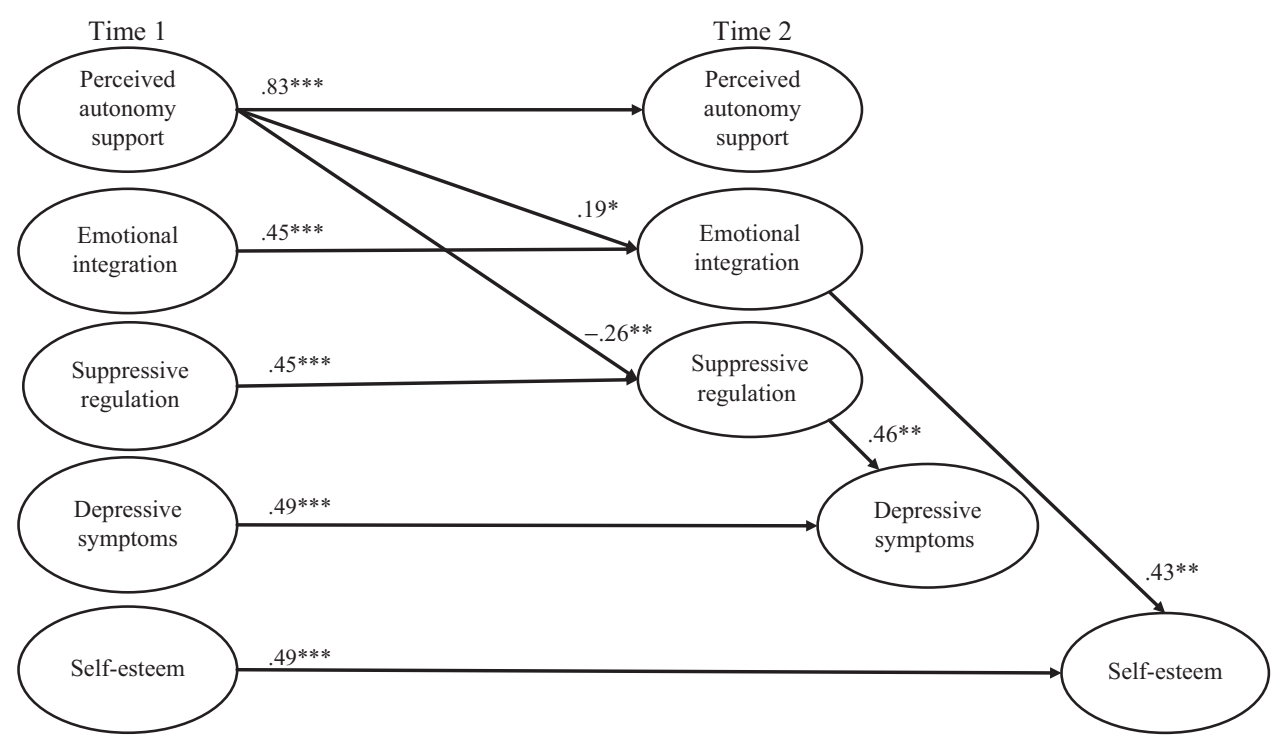

Figure 1. Structural Model of the Associations Among Perceived Maternal Autonomy Support, Emotion Regulation Styles, and Early Adolescents' Well-being.

Notes: Coefficients are standardized path coefficients. Effects of age and within-time correlations are not shown. ${ }^{*} p<.05, * * p<.01, * * * p<.001$.

marginally significant associations found in the previous simple cross-lagged analyses were included in the encompassing models. We did not include emotional dysregulation in these models because no significant paths were found from autonomy support to dysregulation or from dysregulation to the outcomes. As such, dysregulation could not function as an intervening variable of associations between autonomy support and the outcomes.

In the first step, we tested a model in which perceived autonomy-supportive parenting was related to changes in the outcomes only indirectly through changes in the ER styles (see Figure 1). We controlled for stability in all study variables, and within-time correlations were specified between all constructs. The integrated model included cross-lagged paths from autonomy support to integration and suppression. Next, integration and suppression were modeled as predictors of, respectively, self-esteem and depressive symptoms at Time 2 while controlling for baseline levels in these outcomes. This integrated model (see Figure 1) showed adequate fit, $\chi^{2}(398)=565,43, \mathrm{CFI}=.94$, RMSEA $=.04, \mathrm{SRMR}=.07 . .^{1}$ Note that the effect of autonomy-supportive parenting on integration, which in the initial set of models was only marginally significant, now became fully significant.

In the next step, we compared the fit of the initial (full mediation) model with the fit of a partial mediation model in which additional direct paths from perceived autonomy-supportive parenting to both adjustment outcomes were allowed. These direct paths were not significant $(\beta=-.03, p>.10$ for depressive symptoms and $\beta=.06, p>.10$ for self-esteem), and they did not improve model fit, $\Delta \chi^{2}(2)=.28$, $p>.10$. These results support the full mediation model (see Figure 1).

In the third step, we tested the possibility that depressive symptoms (measured at Time 1) would reciprocally affect perceived autonomy support and the use of 


\section{Katrijn Brenning, Bart Soenens, Stijn Van Petegem et al.}

suppression, and that self-esteem (measured at Time 1) would reciprocally affect the use of integration. However, the significant or marginally significant pathways that were found in previous simpler cross-lagged analyses did not remain significant when included in the integrated model $(\beta=-.14, p>.10$ between depressive symptoms and perceived autonomy support; $\beta=.09, p>.10$ between depressive symptoms and suppression; $\beta=.11, p>.10$ between self-esteem and integration), and they did not improve model fit, $\Delta \chi^{2}(3)=3.74, p>.10$.

In the final step, we tested whether the indirect paths from perceived autonomy support to self-esteem and depressive symptoms via, respectively, integration and suppression were significant (Preacher \& Hayes, 2008). The results show that the indirect path from autonomy support to self-esteem through integration was not significant $(\beta=.08, p>.10)$ and that the indirect path from autonomy support to depressive symptoms through suppression was marginally significant $(\beta=-.12$, $p=.06)$. Although all findings pointed in the direction of a mediation model, the indirect effects may not have reached full significance because of the conservative way in which paths were tested (i.e., controlling for initial levels of all variables in the model and for within-time associations).

\section{Discussion}

The central aim of this study was to examine longitudinal associations between perceived autonomy-supportive parenting, different ER styles, and early adolescents' adjustment. Using a cross-lagged longitudinal design, we found that maternal autonomy support predicted increases in adaptive ER (i.e., integration) and decreases in maladaptive ER (i.e., suppression). In turn, these changes in ER were associated with early adolescent adjustment. By contrast, dysregulation was found to predict decreases in autonomy support rather than being predicted by such parenting. In the following paragraphs, we discuss these findings in greater detail.

First, in line with hypotheses derived from SDT and with earlier (mostly crosssectional) research (e.g., Soenens et al., 2007), we found that perceived maternal autonomy support promotes early adolescents' well-being. Specifically, when early adolescents experienced their mothers as autonomy-supportive, they reported decreases in depressive symptoms and increases in self-esteem one year later. These findings are consistent with a handful of other longitudinal studies showing that autonomy support is not only related to adjustment concurrently but is also predictive of changes in children's and adolescents' adjustment, as autonomy support was predictive of children's academic and social skills (Joussemet, Koestner, Lekes, \& Landry, 2005) and of adolescents' depressive symptoms (Van der Giessen, Branje, \& Meeus, 2013). Whereas the present study found the strongest evidence for effects of perceived autonomy-supportive parenting on early adolescent depressive symptoms and selfesteem, there was also a marginally significant effect of depressive symptoms on decreases in autonomy support in the initial cross-lagged models. Previous studies also provided evidence for effects of adolescent adjustment on perceived autonomy support. For example, a recent study with early and middle adolescents showed a longitudinal child effect of depressive symptoms on perceived autonomy support (Van der Giessen et al., 2013).

Second, findings showed that perceived maternal autonomy support predicted an increasing reliance on integration to regulate sadness. Although we also examined the possibility that adolescents who show adaptive ER styles might make it easier for 
parents to be autonomy-supportive, the current findings did not provide evidence for such reciprocal relations. Possibly, it is not so much the use of emotional integration itself that elicits autonomy support but rather adolescents' constructive communication about emotions following from integration. Future research using other time intervals and other research methods (e.g., a diary study) could further investigate this possibility of bidirectional effects. The effect of perceived autonomy support on integration is in line with SDT and with previous cross-sectional findings by Roth and colleagues (Roth \& Assor, 2012; Roth et al., 2009). Because autonomy-supportive parents are expected to be attentive to children's feelings and to display a non-judgmental attitude toward negative emotions, children may experience freedom to explore and express their emotions. Indeed, by parents' displaying a genuine interest in children's emotions, children may learn to take interest in their own negative emotions, thereby using those emotions as guides for future behavior (Dunsmore, Her, Halberstadt, \& Perez-Rivera, 2009). The combination of freedom to experience emotions as they are and to use emotions as a guide for adaptive behavior is precisely what characterizes emotional integrative functioning. Given this reasoning, autonomy-supportive parenting can be expected to relate to emotion-coaching parenting according to metaemotion theory (Gottman et al., 1997) and to supportive parental responses as defined in emotion socialization theory (Fabes et al., 2001). As such, future research may examine whether effects of a general parenting style such as autonomy support are mediated by emotion-specific parenting practices.

By contrast, when parents lack autonomy support, they may minimize, alter, or even deny negative emotions. When experiencing such a lack of autonomy support, children may feel like certain emotions are unacceptable and need to be suppressed. The current study supported this hypothesis by showing that perceived autonomy support predicted a decreased use of suppression, which is also in line with previous cross-sectional research (e.g., Roth \& Assor, 2012). Again, it would be interesting for future research to examine whether autonomy-supportive parenting is related negatively to emotiondismissing parenting (as defined in meta-emotion theory, Gottman et al., 1997) and to minimization or punitive parenting reactions (as defined in emotion socialization theory, Fabes et al., 2001).

By contrast to the significant effects of perceived autonomy support on integration and suppression, we did not find a significant cross-lagged path from autonomy support to dysregulation. Possibly, parenting dimensions other than autonomy support may be involved more strongly in the development of dysregulation. For example, maternal lack of sensitivity (Allhusen et al., 2004) or failure to provide structure and adequate supervision (Frick \& Morris, 2004; Morris et al., 2007) predict children's and adolescents' emotional dysregulation. Indeed, to avoid dysregulation, adolescents need to feel skilled to handle their negative emotions, and parents' provision of guidance and structure may be critical in this regard. Hence, future research on parenting and ER could also include other parenting variables, such as parental sensitivity and structure. Another possibility is that dysregulation would be relatively more strongly determined by temperamental differences (e.g., Orta, Corapci, Yagmurlu, \& Aksan, 2013), a possibility that needs further examination as well.

Although no parent effect on dysregulation was found, a significant child effect from dysregulation to perceived autonomy-supportive parenting was obtained. One possible explanation for this child effect is that dysregulation (by contrast to suppression) represents a relatively visible and manifest failure to cope effectively with emotions. As such, dysregulation might be a direct source of concern for parents. Also, 


\section{Katrijn Brenning, Bart Soenens, Stijn Van Petegem et al.}

dysregulation is likely to manifest in an intensive and chaotic display of negative emotions, which might trigger feelings of helplessness or even irritation in parents. Driven by such negative feelings, parents may become less attuned to their child's perspective and more inclined to use controlling practices to alter children's way of handling emotions (Soenens, Luyckx, Vansteenkiste, Duriez, \& Goossens, 2008).

A third set of relevant findings concerns the intervening role of the ER styles in the associations between perceived autonomy support and the outcomes. We found that autonomy support related to increases in integration and decreases in suppression, which in turn related to increases in self-esteem and depressive symptoms, respectively. Moreover, none of the initially obtained direct longitudinal associations between maternal autonomy support and the outcomes were significant after taking into account the effects of the ER strategies. These findings suggest that processes of ER represent one important pathway through which autonomy-supportive parenting translates into favorable adjustment. These findings contribute to the literature because all evidence for the role of ER in effects of autonomy-supportive parenting to date has been cross-sectional in nature (e.g., Roth et al., 2009). Still, the findings regarding the intervening role of ER should be interpreted with caution because indirect effects were marginally significant at best. Future research should further investigate the mediating role of ER in larger and more heterogeneous samples.

\section{Limitations and Directions for Future Research}

Although this study yielded several novel findings, some important limitations need to be mentioned. First, data were collected in a rather specific way which limits the study's generalizability. That is, data were gathered by undergraduate university students who recruited participants in the context of a course on developmental psychology. Second, there was substantial attrition from Time 1 to Time 2. Although the MCAR test indicated that the data were likely to be missing at random, future longitudinal research (preferably with less attrition) needs to replicate the present results. Third, all variables were assessed through self-report, which may artificially inflate observed relations through shared method variance. It should be noted, however, that the associations were obtained between self-report measures collected one year apart. The reliance on a longitudinal design and conservative testing of the hypotheses through structural equation modeling with latent variables further alleviates the concern of shared method variance. Moreover, there are good theoretical reasons to focus on early adolescents' own perceptions of mothers' autonomy support as it is the degree to which children subjectively experience autonomy support that might be the strongest predictor of their own development. With regard to ER and developmental outcomes, self-report instruments are also seen as the most direct way of tapping into subjective experiences, which is vitally important in research on internalizing problems (Garber \& Kaminski, 2000). Nonetheless, future longitudinal research should use multiple informants and experimental designs to replicate and extend the current results.

Another important direction for future research is to examine whether the current model can be generalized to other emotion types (e.g., anger regulation) and whether it can be generalized to fathers, who also play an important role in children's development of ER (for an overview, see Cassano, Perry-Parrish, \& Zeman, 2007). For instance, fathers are more likely to respond to sadness with minimization whereas mothers are more likely to respond with expressive encouragement and 
problem-focused strategies. Further, previous studies suggest that maternal and paternal parenting may interact to predict children's ER strategies (McElwain, Halberstadt, \& Volling, 2007), and both maternal and paternal autonomy-supportive behaviors are important for child and adolescent well-being and operate through similar mechanisms (e.g., Soenens \& Vansteenkiste, 2005). As such, future studies may want to investigate maternal and paternal parenting simultaneously. As mentioned earlier, future research may also include concepts from different theoretical models, including meta-emotion theory and also attachment theory. For instance, similarities and differences between the SDT-based concept of dysregulation and the attachment theory-based concept of hyperactivation of emotions may be addressed (Cassidy, 1994; Mikulincer, Shaver, \& Pereg, 2003). Whereas dysregulation involves a helpless and unintentional way of handling negative emotions, hyperactivation may be a relatively more intentional style of ER aimed at eliciting parental or peer attention.

\section{Conclusion}

Together with the relatively modest stability coefficients for the ER styles, the observation that perceived autonomy support predicts changes in these styles during pre- and early adolescence testifies to the fact that these styles are dynamic and susceptible to change during this developmental period. Indeed, plenty of evidence suggests that there is still much room for improvement in ER capacities, that early adolescence represents a particularly challenging period for ER, and that ER styles are in flux (Morris et al., 2007; Zeman et al., 2006). Perceived maternal autonomy support appears to represent a critical resource to navigate children through this developmental period. When mothers are perceived as autonomy-supportive, children increasingly engage in emotional integration and decrease their use of suppressive regulation. These changes in ER, in turn, contribute to early adolescents' adjustment.

In terms of clinical implications, these findings suggest that both autonomysupportive parenting behaviors and ER styles may represent targets for therapeutic intervention. Although several parenting programs have been identified, many of these programs target the role of involvement, warmth, and responsiveness, yet do not explicitly address the role of autonomy support (but see Joussemet, Mageau, \& Koestner, 2014 for an exception). The role of ER is well recognized in several intervention programs as well. For example, one of the main treatment goals of dialectical behavior therapy (Linehan, 1998) is to teach skills involved in adequate ER. Ultimately, the current research seems to suggest that both parenting and ER are important targets for adequate prevention and intervention, as improvements in the quality of ER styles may be short-lived when parenting behaviors are not changed as well.

\section{References}

Allhusen, V., Belsky, J., Booth, C. L., Bradley, R., Brownell, C. A., Burchinal, M., et al. (2004). Affect dysregulation in the mother-child relationship in the toddler years: Antecedents and consequences. Development and Psychopathology, 16, 43-68. doi: 10.1017/ s0954579404040404

Brenning, K., \& Braet, C. (2013). The emotion regulation model of attachment: An emotionspecific approach. Personal Relationships, 20, 107-123. doi: 10.1111/j.1475-6811.2012 $.01399 . x$

Brenning, K., Soenens, B., Braet, C., \& Bosmans, G. (2012). Attachment and depressive symptoms in middle childhood and early adolescence: Testing the validity of the emotion 


\section{Katrijn Brenning, Bart Soenens, Stijn Van Petegem et al.}

regulation model of attachment. Personal Relationships, 19, 445-464. doi: 10.1111/j.1475 $-6811.2011 .01372 . x$

Cassano, M., Perry-Parrish, C., \& Zeman, J. (2007). Influence of gender on parental socialization of children's sadness regulation. Social Development, 16, 210-231. doi: 10.1111/j.14679507.2007.00381.x

Cassidy, J. (1994). Emotion regulation: Influences of attachment relationships. In N. A. Fox (Ed.), The development of emotion regulation: Biological and behavioral considerations (Vol. 59), Monographs of the Society for Research in Child Development. 59, 228-250.

Cicchetti, D., Ackerman, B. P., \& Izard, C. E. (1995). Emotions and emotion regulation in developmental psychopathology. Development and Psychopathology, 7, 1-10.

Deci, E. L., \& Ryan, R. M. (2000). The 'what' and 'why' of goal pursuits: Human needs and the self-determination of behavior. Psychological Inquiry, 11, 227-268. doi: 10.1207/ s15327965pli1104_01

Dunsmore, J. C., Booker, J. A., \& Ollendick, T. H. (2013). Parental emotion coaching and child emotion regulation as protective factors for children with oppositional defiant disorder. Social Development, 22, 444-466. doi: 10.1111/j.1467-9507.2011.00652.x

Dunsmore, J. C., Her, P., Halberstadt, A. G., \& Perez-Rivera, M. B. (2009). Parents' beliefs about emotions and children's recognition of parents' emotions. Journal of Nonverbal Behavior, 33, 121-140. doi: 10.1007/s10919-008-0066-6

Fabes, R. A., Leonard, S. A., Kupanoff, K., \& Martin, C. L. (2001). Parental coping with children's negative emotions: Relations with children's emotional and social responding. Child Development, 72, 907-920. doi: 10.1111/1467-8624.00323

Frick, P. J., \& Morris, A. S. (2004). Temperament and developmental pathways to conduct problems. Journal of Clinical Child and Adolescent Psychology, 33, 54-68. doi: 10.1207/ s15374424jccp3301_6

Garber, J., \& Kaminski, K. M. (2000). Laboratory and performance-based measures of depression in children and adolescents. Journal of Clinical Child Psychology, 29, 509-525. doi: 10.1207/s15374424jccp2904_5

Gottman, J. M., Katz, L. F., \& Hooven, C. (1997). Meta-emotion: How families communicate emotionally. Hillsdale, NJ: Lawrence Erlbaum Associates.

Grolnick, W. S., Ryan, R. M., \& Deci, E. L. (1991). Inner resources for school-achievement: Motivational mediators of children's perceptions of their parents. Journal of Educational Psychology, 83, 508-517. doi: 10.1037/0022-0663.83.4.508

Gross, J. J. (2013). Emotion regulation: Taking stock and moving forward. Emotion (Washington, D.C.), 13, 359-365. doi: 10.1037/a0032135

Harter, S. (1985). Manual for the self-perception profile for children. University of Denver, Denver, CO.

Joussemet, M., Koestner, R., Lekes, N., \& Landry, R. (2005). A longitudinal study of the relationship of maternal autonomy support to children's adjustment and achievement in school. Journal of Personality, 73, 1215-1235. doi: 10.1111/j.1467-6494.2005.00347.x

Joussemet, M., Landry, R., \& Koestner, R. (2008). A self-determination theory perspective on parenting. Canadian Psychology-Psychologie Canadienne, 49, 194-200. doi: 10.1037/ a0012754

Joussemet, M., Mageau, G. A., \& Koestner, R. (2014). Promoting optimal parenting and children's mental health: A preliminary evaluation of the how-to parenting program. Journal of Child and Family Studies, 23, 949-964. doi: 10.1007/s10826-013-9751-0

Kessler, R. C., Avenevoli, S., \& Merikangas, K. R. (2001). Mood disorders in children and adolescents: An epidemiologic perspective. Biological Psychiatry, 49, 1002-1014. doi: 10.1016/s0006-3223(01)01129-5

Kline, R. B. (2010). Principles and practice of structural equation modeling (3rd ed.). New York: Guilford Press.

Kovacs, M. (1985). The children's depression inventory (CDI). Psychopharmacology Bulletin, 21, 995-998.

Larson, R. W., Moneta, G., Richards, M. H., \& Wilson, S. (2002). Continuity, stability, and change in daily emotional experience across adolescence. Child Development, 73, 11511165. doi: 10.1111/1467-8624.00464

Linehan, M. M. (1998). An illustration of dialectical behavior therapy. Session-Psychotherapy in Practice, 4, 21-44. 
Little, R. J. A., \& Rubin, D. B. (1987). Statistical analysis with missing data. New York: Wiley.

Matte-Gagne, C., Bernier, A., \& Gagne, C. (2013). Stability of maternal autonomy support between infancy and preschool age. Social Development, 22, 427-443. doi: 10.1111/j.14679507.2012.00667.x

McElwain, N. L., Halberstadt, A. G., \& Volling, B. L. (2007). Mother- and father-reported reactions to children's negative emotions: Relations to young children's emotional understanding and friendship quality. Child Development, 78, 1407-1425. doi: 10.1111/j.14678624.2007.01074.x

McRae, K., Gross, J. J., Weber, J., Robertson, E. R., Sokol-Hessner, P., Ray, R. D., et al. (2012). The development of emotion regulation: An fMRI study of cognitive reappraisal in children, adolescents and young adults. Social Cognitive and Affective Neuroscience, 7, 11-22. doi: $10.1093 / \mathrm{scan} / \mathrm{nsr} 093$

Mikulincer, M., Shaver, P. R., \& Pereg, D. (2003). Attachment theory and affect regulation: The dynamics, development, and cognitive consequences of attachment-related strategies. Motivation and Emotion, 27, 77-102. doi: 10.1023/a:1024515519160

Morris, A. S., Silk, J. S., Steinberg, L., Myers, S. S., \& Robinson, L. R. (2007). The role of the family context in the development of emotion regulation. Social Development, 16, 361-388. doi: $10.1111 /$ j.1467-9507.2007.00389.x

Muthén, L. K., \& Muthén, B. O. (2012). Mplus user's guide (Vol. 7). Los Angeles, CA: Muthén \& Muthén.

Orta, I. M., Corapci, F., Yagmurlu, B., \& Aksan, N. (2013). The mediational role of effortful control and emotional dysregulation in the link between maternal responsiveness and Turkish preschoolers' social competency and externalizing symptoms. Infant and Child Development, 22, 459-479. doi: 10.1002/icd.1806

Preacher, K. J., \& Hayes, A. F. (2008). Asymptotic and resampling methods for estimating and comparing indirect effects. Behavior Research Methods, 40, 879-891.

Romeo, R. D. (2013). The teenage brain: The stress response and the adolescent brain. Current Directions in Psychological Science, 22, 140-145. doi: 10.1177/0963721413475445

Roth, G., \& Assor, A. (2012). The costs of parental pressure to express emotions: Conditional regard and autonomy support as predictors of emotion regulation and intimacy. Journal of Adolescence, 35, 799-808. doi: 10.1016/j.adolescence.2011.11.005

Roth, G., Assor, A., Niemiec, C. P., Ryan, R. A., \& Deci, E. L. (2009). The emotional and academic consequences of parental conditional regard: Comparing conditional positive regard, conditional negative regard, and autonomy support as parenting practices. Developmental Psychology, 45, 1119-1142. doi: 10.1037/a0015272

Soenens, B., Luyckx, K., Vansteenkiste, M., Duriez, B., \& Goossens, L. (2008). Clarifying the link between perceived parental psychological control and adolescents' depressive feelings: Reciprocal versus unidirectional models of influence. Merrill-Palmer Quarterly, 54, 411444.

Soenens, B., \& Vansteenkiste, M. (2005). Antecedents and outcomes of self-determination in 3 life domains: The role of parents' and teachers' autonomy support. Journal of Youth and Adolescence, 34, 589-604. doi: 10.1007/s10964-005-8948-y

Soenens, B., \& Vansteenkiste, M. (2010). A theoretical upgrade of the concept of parental psychological control: Proposing new insights on the basis of self-determination theory. Developmental Review, 30, 74-99. doi: 10.1016/j.dr.2009.11.001

Soenens, B., Vansteenkiste, M., Lens, W., Luyckx, K., Goossens, L., Beyers, W., et al. (2007). Conceptualizing parental autonomy support: Adolescent perceptions of promotion of independence versus promotion of volitional functioning. Developmental Psychology, 43, 633646. doi: 10.1037/0012-1649.43.3.633

Timbremont, B., Braet, C., \& Roelofs, J. (2008). Children's depression inventory, Nederlandstalige versie (children's depression inventory, Dutch version). Amsterdam: Pearson Assessment and Information.

Van der Giessen, D., Branje, S., \& Meeus, W. (2013). Perceived autonomy support from parents and best friends: Longitudinal associations with adolescents' depressive symptoms. Social Development, 23, 537-555.

Wichstrom, L. (1995). Harters' self-perception profile for adolescents: Reliability, validity, and evaluation of the question format. Journal of Personality Assessment, 65, 100-116. doi: 10.1207/s15327752jpa6501_8 


\section{Katrijn Brenning, Bart Soenens, Stijn Van Petegem et al.}

Zeman, J., Cassano, M., Perry-Parrish, C., \& Stegall, S. (2006). Emotion regulation in children and adolescents. Journal of Developmental and Behavioral Pediatrics, 27, 155-168. doi: 10.1097/00004703-200604000-00014

\section{Note}

1. To examine the moderating role of age, a multigroup analysis was conducted, comparing a constrained model in which the structural paths were set to be invariant across the youngest (9-11 years) and the oldest groups (12-14 years) with an unconstrained model in which these parameters were freely estimated across groups. No significant differences were found between the two groups, $\Delta \operatorname{chi}^{2}(9)=11.97$, $p>.05$. 